\title{
1. Quantification of Cholesterol Solubilized in Dietary Micelles: 2Dependence on Human Bile Salt Variability and the Presence of ${ }_{3}$ Dietary Food Ingredients
}

${ }_{4}$ Filipe M. Coreta-Gomes, ${ }^{\dagger, \S}$ Winchil L. C. Vaz, ${ }^{\dagger, \|}$ Emeric Wasielewski, ${ }^{\S}$ Carlos F. G. Geraldes, ${ }^{*}, \S$

$s$ and Maria João Moreno ${ }^{*}, \dagger, \S$

$6{ }^{\dagger}$ Chemistry Department, University of Coimbra Rua Larga, Largo D. Dinis, 3004-535 Coimbra, Portugal

7 "Department of Life Sciences, Faculty of Science and Technology, University of Coimbra 3000-393 Coimbra, Portugal

$8{ }^{\S}$ Coimbra Chemistry Center, CQC, Rua Larga, University of Coimbra, 3004-535 Coimbra, Portugal

9 "CEDOC, NOVA Medical School, Faculdade de Ciências Médicas, Universidade Nova de Lisboa, Lisbon, Portugal

11 ABSTRACT: The solubility of cholesterol in bile salt (BS) micelles is 12 important to understand the availability of cholesterol for absorption in the 13 intestinal epithelium and to develop strategies to decrease cholesterol 14 intake from the intestinal lumen. This has been the subject of intense 15 investigation, due to the established relation between the development of 16 diseases such as atherosclerosis and high levels of cholesterol in the blood.

17 In this work we quantify the effect of BS variability on the amount of 18 cholesterol solubilized. The effect of some known hypocholesterolemic 19 agents usually found in the diet is also evaluated, as well as some insight 20 regarding the mechanisms involved. The results show that, depending on 21 the bile salt composition, the average value of sterol per micelle is equal to 22 or lower than 1 . The amount of cholesterol solubilized in the BS micelles is 23 essentially equal to its total concentration until the solubility limit is 24 reached. Altogether, this indicates that the maximum cholesterol solubility

25 in the BS micellar solution is the result of saturation of the aqueous phase and depends on the partition coefficient of cholesterol 26 between the aqueous phase and the micellar pseudophase. The effect on cholesterol maximum solubility for several food 27 ingredients usually encountered in the diet was characterized using methodology developed recently by us. This method allows 28 the simultaneous quantification of both cholesterol and food ingredient solubilized in the BS micelles even in the presence of 29 larger aggregates, therefore avoiding their physical separation with possible impacts on the overall equilibrium. The phytosterols 30 stigmasterol and stigmastanol significantly decreased cholesterol solubility with a concomitant reduction in the total amount of 31 sterol solubilized, most pronounced for stigmasterol. Those results point toward coprecipitation being the major cause for the 32 decrease in cholesterol solubilization by the BS micelles. The presence of tocopherol and oleic acid leads to a small decrease in 33 the amount of cholesterol solubilized while palmitic acid slightly increases the solubility of cholesterol. Those dietary food 34 ingredients are completely solubilized by the BS micelles, indicating that the effects on cholesterol solubility are due to changes in 35 the properties of the mixed micelles.

37 Of the $1800 \mathrm{mg}$ of cholesterol absorbed per day by an average 38 human, $600 \mathrm{mg}$ are supplied by the diet, and the remaining is 39 due to endogenous production. ${ }^{1}$ Although sterols are vital 40 molecules for eukaryotic life forms, including humans, 41 cholesterol is also known for its potentially lethal properties. 42 Indeed, high cholesterol levels in blood are supposed to be 43 responsible for atherosclerosis, one of the most deadly and 44 incapacitating diseases in humans. ${ }^{2}$ It is therefore crucial to 45 develop methods that may reduce cholesterol blood level. The 46 solubilization of cholesterol in the intestine, which is strongly 47 dependent on the diet, can be a key research target for this task. 48 Given its low solubility in water, ${ }^{3}$ cholesterol needs to be solubilized prior to absorption. This is achieved with the help of 49 several molecules present in the duodenum content, namely 50 bile salts (BS), the most representative of which are the ones 51 conjugated with glycine, phospholipids (PL), and fatty acids 52 (FA). This mixture of components self-aggregates and forms 53 either intermixed micelles or vesicles depending on the BS/ 54 lipid ratio. ${ }^{4}$ These structures solubilize cholesterol and other 55 hydrophobic nutrients and carry them to the apical membrane 56 of the brush border located in the intestinal epithelium. ${ }^{5-7} \quad 57$

Received: February 24, 2016

Revised: April 14, 2016 



Palmitic acid

Oleic acid

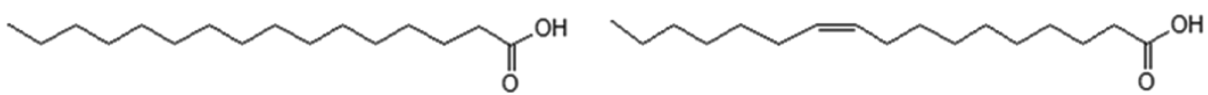

Figure 1. Molecular structures of BS (GDCA, GCDCA and GCA), cholesterol (enriched in ${ }^{13} \mathrm{C}$ in position 4), phytosterols (stigmastanol and stigmasterol), vitamin ( $\alpha$-tocopherol) and FA (saturated - palmitic acid; unsaturated - oleic acid).

58 BS are crucial for cholesterol absorption. ${ }^{4-6,8}$ Cholesterol 59 solubilization by BS has been extensively studied in the past, 60 focusing mainly on the therapeutic potentialities for gallstone 61 disease. $^{9-11}$ A substantial mapping of ternary and binary phase 62 diagrams of PL, BS, and cholesterol was determined. ${ }^{4,11-15}$ The 63 size of the micelles, mixed micelles, and vesicles formed with 64 the different proportions of these components as well as 65 cholesterol solubility has been addressed. ${ }^{9,12-29}$ One important 66 observation was that BS with distinct hydrophobicities have 67 different capacities to solubilize and to deliver cholesterol to 68 membranes. However, the delivery efficiency is not directly 69 dependent on the amount of cholesterol solubilized by the 70 micelles. This has been interpreted as delivery being through 71 aqueous cholesterol monomer and not by the fusion of 72 cholesterol loaded micelles and the membranes. ${ }^{6}$ Another 73 important factor in the relation between solubilization of 74 cholesterol in BS micelles and its delivery to membranes may 75 be related to the interaction of BS with the lipid bilayers and 76 the effects on the properties of the latter. $6,30-33$

77 Most work has been done with taurine conjugates, although 78 glycine-conjugated BS have a higher prevalence in humans. ${ }^{34}$ 79 This apparent contradiction is due to the lower $\mathrm{p} K_{\mathrm{a}}$ value of the 80 taurine conjugates, ${ }^{35}$ with the ionized species being the only 81 relevant one in the whole range of $\mathrm{pH}$ values found in the 82 duodenum (6 to 8$).{ }^{18}$ Another limitation associated with most 83 previous work is the need to physically separate the distinct 84 components in the system (micelles, vesicles, and/or crystals). 85 We have developed a method to quantitatively follow the 86 solubilization of cholesterol in $\mathrm{BS}$ micelles by ${ }^{13} \mathrm{C} \mathrm{NMR}$ spectroscopy using $\left[4{ }^{13} \mathrm{C}\right]$-cholesterol. ${ }^{19}$ The main advantage 87 of this method is that it permits monitoring cholesterol 88 solubilization in the micelle in a noninvasive way, i.e., without 89 using any technique that could disrupt the equilibrium, such as 90 filtration or chromatography.

In this work we extended the application of the NMR 92 method developed to quantitatively evaluate the dependence of 93 cholesterol solubilization in BS micelles upon the variation of 94 some conditions present in the duodenum. Our approach was 95 to reduce the complexity of the system (duodenum content) to 96 two major class components only (BS and cholesterol). First 97 we addressed the possibility of synergistic effects between the 98 most representative BS present in the duodenum and how they 99 affect cholesterol solubilization. Different compositions of BS, 100 namely single, binary, and a physiologically relevant ternary 101 composition were studied at the concentrations usually 102 observed in the duodenum. These studies provide valuable 103 information regarding the effect of BS variability on cholesterol 104 solubilization in the intestinal lumen, and therefore on its 105 absorption through the intestinal epithelium.

106

One of the most used strategies to reduce cholesterol 107 absorption in the intestine is to include sterols of vegetable 108 origin in the diet. $^{36,37}$ Phytosterols are known to reduce 109 cholesterol solubilization in BS micelles and in this way 110 decrease cholesterol absorption. ${ }^{22,26,37-42}$ The mechanism 111 involved in the reduction of cholesterol solubilization by the 112 BS micelles is not clear, and may be the result of a decrease in 113 cholesterol solubility in the micellar phase (due to cosolubiliza- 114 tion) and/or a reduction of cholesterol solubility in the aqueous 115 
116 phase (due to coprecipitation). The distinction between the 117 two mechanisms is relevant because a reduction in the capacity 118 of BS micelles to solubilize hydrophobic solutes may 119 compromise the absorption of important dietary compounds 120 such as lipophilic vitamins. Important insight regarding the 121 mechanism involved may be obtained from the quantification 122 of both cholesterol and phytosterol solubilized in the same BS 123 micellar solution. The method used in this work permits the 124 direct quantification of solubilized cholesterol (due to enrich125 ment in ${ }^{13} \mathrm{C}$ at carbon 4) as well as phytosterol (from the 126 chemical shift induced in the BS resonances). ${ }^{19}$ The 127 phytosterols studied in this work are stigmasterol and 128 stigmastanol, which are representative of the two most 129 abundant classes known to affect cholesterol solubility.

130 Fatty acids are another important class of dietary compounds 131 known to affect cholesterol absorption. ${ }^{43}$ In this regard we 132 addressed also the quantitative effect of the saturated FA 133 palmitic acid (PA) and the unsaturated FA oleic acid (OA) 134 upon cholesterol solubilization by BS micelles. Finally we also 135 studied the effect of $\alpha$-tocopherol (the component of vitamin $136 \mathrm{E}$ ) on the solubilization of cholesterol as a model of a lipophilic 137 vitamin. Figure 1 shows the structures of the different BS and 138 dietary phytochemicals used in this work.

\section{EXPERIMENTAL SECTION}

140 N-Phenyl-1-naphthylamine (NPN) was obtained from Merck 141 (Hohenbrunn, Germany), $\left[4-{ }^{13} \mathrm{C}\right]$ cholesterol and deuterium oxide 142 (99.8\%) for NMR experiments were obtained from CortecNet (Paris, 143 France), and the BS sodium glycochenodeoxycholate (GCDCA), 144 sodium glycocholate (GCA), sodium glycodeoxycholate (GDCA), $\alpha$ 145 tocopherol, stigmastanol and stigmasterol were obtained from Sigma 146 (Steinheim, Germany), while oleic acid and palmitic acid were 147 provided by Avanti. The nonaqueous solvents used for sample 148 preparation (chloroform, methanol, and acetone) were of spectrosco149 py grade, and the aqueous buffer components (Tris- $\mathrm{HCl}, \mathrm{NaCl}$, and $150 \mathrm{NaN}_{3}$ ) were of high purity and purchased from Sigma; water was first 151 distilled and further purified by activated charcoal and deionization.

152 The critical micellar concentration (CMC) of single, binary, and 153 ternary BS mixtures used were determined using a method described 154 in the literature, ${ }^{44}$ based on the increase in fluorescence quantum yield 155 and blue shift of the fluorescence emission spectra of $\mathrm{N}$-phenyl-1156 naphthylamine (NPN) upon association with preformed micelles. The 157 total concentration of NPN was $1 \mu \mathrm{M}$, and the concentrations of 158 simple, binary and ternary BS mixtures in the aqueous buffer were 159 changed between 0 and $50 \mathrm{mM}$ at $37^{\circ} \mathrm{C}$. The fluorescent emission of 160 NPN was followed using an excitation wavelength of $330 \mathrm{~nm}$ on a 161 Cary Eclipse fluorescence spectrophotometer from Varian (Victoria, 162 Australia) equipped with a thermostated multicell holder accessory. 163 Tocopherol solubility was followed by absorption spectroscopy using 164 its molar absorptivity coefficient $\left(3.2 \times 10^{3} \mathrm{~cm}^{-1} \mathrm{M}^{-1}\right.$ at $\left.\lambda=292 \mathrm{~nm}\right)$. 165 The concentration of tocopherol solubilized in GDCA micelles was 166 obtained from its absorption at $292 \mathrm{~nm}$, using a path length $0.1 \mathrm{~cm}$. 167 The size of BS micelles at a total concentration of $50 \mathrm{mM}$, with and 168 without cholesterol solubilized, was also characterized by dynamic light 169 scattering (DLS) using a Beckman Coulter N4 Plus apparatus 170 operating with a $\mathrm{He}$ neon laser at $632.8 \mathrm{~nm}$ as light source and a 171 scattering angle of $90^{\circ}$. The autocorrelation curves were analyzed with 172 the PSC control software provided with the equipment. The refraction 173 index considered for the samples was 1.33 , and the viscosity was 1.127 $174 \mathrm{cP}^{45}$ The experiments were done at $37{ }^{\circ} \mathrm{C}$, up to six measurements 175 were run for each sample, and two independent samples were 176 analyzed.

177 Proton-decoupled ${ }^{13} \mathrm{C}$ NMR spectra acquisition was performed on a 178 Varian VNMRS 600 NMR spectrometer equipped with a high field 179 "switchable" $=$ broadband $5 \mathrm{~mm}$ probe with z-gradient operating at a 180 frequency for ${ }^{1} \mathrm{H}$ spectra $(599.72 \mathrm{MHz})$ and for ${ }^{13} \mathrm{C}$ spectra $(150.8$ $181 \mathrm{MHz}) .{ }^{13} \mathrm{C}$ NMR spectra were acquired at $37{ }^{\circ} \mathrm{C}$ with a $90^{\circ}$ pulse angle sequence, a spectral width of $31250 \mathrm{~Hz}$ with an acquisition time 182 of $2.3 \mathrm{~s}$, a relaxation delay of $7 \mathrm{~s}$, and 1024 acquisition scans. Proton 183 decoupling was achieved using a WALTZ-16 decoupling sequence. 184 ${ }^{13} \mathrm{C}-\left\{{ }^{1} \mathrm{H}\right\}$ nuclear Overhauser enhancement (NOE) was obtained by 185 comparing ${ }^{13} \mathrm{C}$ spectra with full proton decoupling and ${ }^{13} \mathrm{C}$ spectra 186 with proton decoupling only during acquisition (spectra acquired with 187 3000 scans). The values used for correcting the NOE effect upon 188 signal intensity are shown in Table S1 and Table S2 (Supporting 189 Information). Spectra were processed with MestreNova 6.1.1 190 (Mestrelab Research, Santiago de Compostela, Spain). The relaxation 191 delay used ensured that the ${ }^{13} \mathrm{C}$ signals are quantitative for carbons 192 with a relaxation time equal to $1.4 \mathrm{~s}$ or shorter. This is the case for $\mathrm{C}_{4} 193$ of cholesterol and for all BS carbons except carbonyl and quaternary 194 carbon atoms. ${ }^{19}{ }^{1} \mathrm{H}$ NMR spectra of stigmasterol and oleic acid were 195 obtained at $37^{\circ} \mathrm{C}$ with a $90^{\circ}$ pulse angle sequence, a spectral width of 196 $7225 \mathrm{~Hz}$, acquisition time of $1.5 \mathrm{~s}$, a relaxation delay of $2.5 \mathrm{~s}$, and 512197 acquisition scans.

198

Aqueous suspensions of $\left[4-{ }^{13} \mathrm{C}\right]$ cholesterol with or without different 199 competitors (phytosterols, FA, tocopherol) were prepared by 200 evaporating the required volume of a solution in chloroform/methanol 201 $(87: 13, \mathrm{v} / \mathrm{v})$, blowing dry nitrogen over the heated solution, and 202 removing traces of organic solvent in a vacuum desiccator for $30 \mathrm{~min} 203$ at $23{ }^{\circ} \mathrm{C}$. The glass tube with the dry residue was then transferred to a 204 thermostatic bath at $37^{\circ} \mathrm{C}$ and hydrated with a preheated solution of 205 $\mathrm{BS}$ in the aqueous buffer (10 mM Tris- $\mathrm{HCl}$ (pH 7.4), $0.15 \mathrm{M} \mathrm{NaCl}, 1206$ $\mathrm{mM}$ ethylenediaminetetraacetic acid (EDTA), and $0.02 \% \mathrm{NaN}_{3}$ in 207 $\mathrm{D}_{2} \mathrm{O}$ ) and was continuously stirred at $100 \mathrm{rpm}$ for 24 to $48 \mathrm{~h}$. In the 208 case of FA, another assay was done by solubilizing first the FA in the 209 BS solution, waiting $24 \mathrm{~h}$, and then adding to a film of cholesterol. The 210 total concentration of BS plus sterol was maintained at $50 \mathrm{mM}$ in all 211 solubilization experiments. In the competition experiments using 212 phytosterols, FA, and tocopherol, the total concentration of GDCA 213 used was $50 \mathrm{mM}$. The samples were left in the bath with continuous 214 stirring during 24 to $48 \mathrm{~h}$ (experiments on the maximum 215 solubilization) and then characterized by ${ }^{13} \mathrm{C}$ NMR to obtain the 216

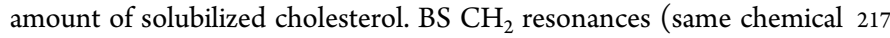
group followed in cholesterol) or TSP (trimethylsilyl propionate) were 218 used as internal standards. NMR assignments were done based on 219 information available in the literature. ${ }^{46}$

220

For the studies of cholesterol solubilization in the bile salt micelles, 221 the total concentration was kept constant $(B S+$ Cholesterol $=50222$ $\mathrm{mM}$ ) while changing the proportion between cholesterol and BS. 223 However, in the competition experiments, this approach leads to a 224 significant decrease in the bile salt concentration that would 225 complicate the analysis of the results. We have therefore opted to 226 maintain the BS concentration at $50 \mathrm{mM}$ in those studies.

\section{RESULTS AND DISCUSSION}

Determination of the CMC of Glycine-Conjugated BS. 229 The CMC of GDCA was characterized previously using two 230 independent methods, isothermal calorimetric titration and dye 231 solubilization (NPN). ${ }^{19}$ The results obtained agreed within the 232 experimental error.

233

In this work we have determined the CMC of some 234 additional BS common in human bile, both simple (GCA and 235 GCDCA), binary mixtures (GCA/GDCA, GCA/GCDCA and 236 GDCA/GCDCA, at equimolar concentrations) and a physio- 237 logically relevant ternary mixture (GCA/GCDCA/GDCA, at 238 the molar ratio $37.5: 37.5: 25)$, using the NPN solubilization 239 method. The results obtained are shown in Figure 2, and the $240 \mathrm{f2}$ calculated CMC values are presented in Table $1 . \quad 241 \mathrm{t}$

The results obtained show that GCA, a BS with three 242 hydroxyl groups, has a much higher CMC than the other two 243 BS studied (GCDCA and GDCA) with two hydroxyl groups 244 (Figure 1), whose CMCs are identical within the experimental 245 error. Thus, the trihydroxy BS is soluble in water in the 246 


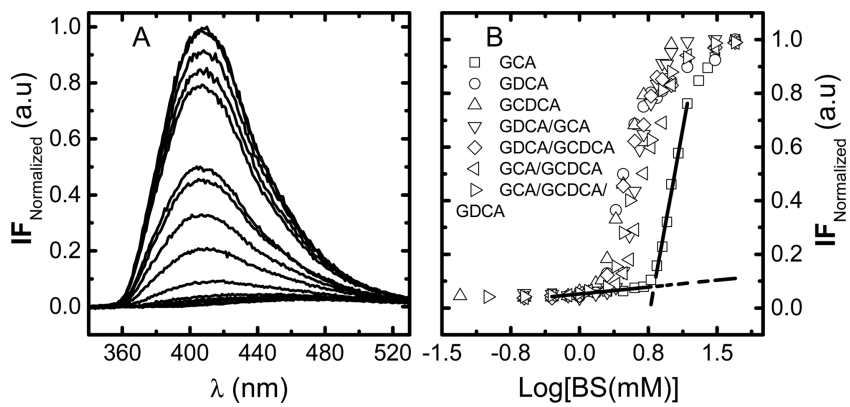

Figure 2. (A) Fluorescent emission spectra of $1 \mu \mathrm{M}$ NPN at increasing concentrations (ranging from 0.25 to $50 \mathrm{mM}$ ) of a ternary GCA/ GCDCA/GDCA (37.5:37.5:25) BS solution in aqueous buffer $\mathrm{pH}=$ 7.4 and $I=150 \mathrm{mM}$ at $37^{\circ} \mathrm{C}$ (excitation wavelength of $330 \mathrm{~nm}$ ). (B) Fluorescence intensity area from 340 to $530 \mathrm{~nm}$ for the different concentrations of $\mathrm{BS}$ mixtures tested. The CMC is calculated from the intersection of the extrapolated fit of the two different regimes, as shown for the case of GCA. The values obtained for the CMC and their standard deviations are shown in Table 1.

247 monomeric state up to a higher concentration than the others, 248 in qualitative agreement with data available in the literature. ${ }^{18,26}$ 249 The CMC values obtained in this work for the isolated BS 250 are systematically smaller than those reported in the literature 251 using ITC for similar conditions (GDCA, $2.0 \mathrm{mM}$, GCDCA, $2522.2 \mathrm{mM}$, and GCA, $8.5 \mathrm{mM}){ }^{26}$ The observed differences 253 between the two data sets are a consequence of the different 254 approaches used to calculate the CMC value from the 255 experimental results. The $\mathrm{CMC}$ is, by definition, the 256 concentration at which micelles start to form (http:// 257 goldbook.iupac.org/C01395.html, accessed on 2016 February 258 4). Accordingly, the CMC values reported in Table 1 were 259 calculated from the intercept between the linear best fits to the 260 measured property at low concentrations and when the 261 property starts changing abruptly due to the formation of 262 micelles (Figure 2B).

263 The CMC values obtained for the binary mixtures, GDCA/ 264 GCA (50:50), GCA/GCDCA (50:50) and GDCA/GCDCA 265 (50:50), were equal to the values predicted from ideal 266 miscibility of surfactants in the micelles, i.e., the free energy 267 associated with the mixing is equal to the sum of free energies 268 of each surfactant. ${ }^{47}$ The CMC obtained for the ternary mixture 269 GCA/GCDCA/GDCA (37.5:37.5:25) is somewhat smaller 270 than the value predicted considering an ideal mixture. This 271 indicates that there are interactions of the components in this
BS mixture, which cannot be described by the average behavior 272 of each BS.

Dependence of Cholesterol Solubilization Index on 274 the Composition of Bile Salt Micelles. The determination 275 of sterol solubilized in the different BS micelles was obtained 276 using the NMR method developed by us. ${ }^{19}$ Briefly, labeled 277 $\left[4-{ }^{13} \mathrm{C}\right]$ cholesterol solubilized in the micelles was followed by 278 ${ }^{13} \mathrm{C}$ NMR spectroscopy directly through the $\left[4-{ }^{13} \mathrm{C}\right]$ cholesterol 279 signal and also by the chemical shift induced in the BS 280 resonances that were most sensitive to the presence of 281 cholesterol. In the chemical shift analysis, the concentration 282 of cholesterol in the micelles was quantified from the chemical 283 shift observed in some selected BS resonances using the slope 284 obtained as a function of cholesterol concentration for the 285 concentration region where this was known (total solute 286 solubilization). The BS resonances were selected based on the 287 sensitivity to the presence of cholesterol and on the linearity 288 observed between the chemical shift and cholesterol concen- 289 tration $\left(R^{2}>0.98\right)$. Figure 3 shows the results obtained for the $290 \mathrm{f} 3$ cholesterol saturation index (CSI) in the distinct mixtures, 291 while the average values and standard deviations are shown in 292 Table 1.

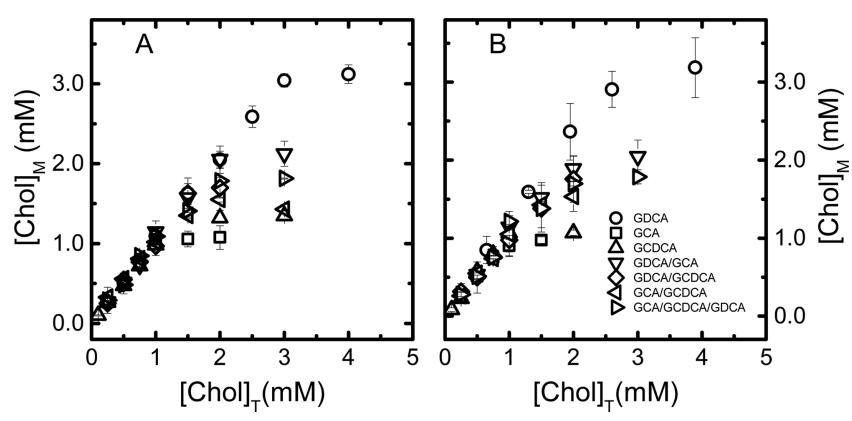

Figure 3. Concentration of $\left[4-{ }^{13} \mathrm{C}\right]$ cholesterol solubilized by $\mathrm{BS}$ micelles $\left([\mathrm{Chol}]_{\mathrm{M}}\right)$ as a function of the total concentrations of cholesterol $\left([\mathrm{Chol}]_{\mathrm{T}}\right)$ for a total concentration of lipid $(\mathrm{BS}+\mathrm{Chol})$ equal to $50 \mathrm{mM}$. The experiments were performed at $37{ }^{\circ} \mathrm{C}$. The amount of solubilized cholesterol was obtained from the area of its resonance for $\left[4{ }^{13} \mathrm{C}\right]$ cholesterol (plot A) or from the chemical shift observed for the bile salt resonances (plot B). The CSI value can be obtained from the maximum $[\mathrm{Chol}]_{\mathrm{M}}$, or from the intersection of the best fit to both regimes in cholesterol solubilization. The average and standard errors associated with the CSI obtained from at least three independent experiments are shown in Table 1.

Table 1. Values of CSI and CMC Determined for Single, Binary and Ternary Mixtures of BS

\begin{tabular}{|c|c|c|c|c|c|c|c|c|}
\hline BS compositions & $\mathrm{CMC}(\mathrm{mM})$ & $\mathrm{CMC}^{b}(\mathrm{mM})$ & CSI (mM) & $\mathrm{CSI}^{c}(\mathrm{mM})$ & radius $(\mathrm{nm})$ & $\overline{\mathrm{A}}^{d}$ & {$[$ Micelles $](\mathrm{mM})$} & $\frac{[\mathrm{Chol}]_{\mathrm{M}}}{[\text { Micelles }]}$ \\
\hline GDCA & $1.7 \pm 0.1$ & & $3.0 \pm 0.1$ & & $2.6 \pm 0.2$ & 16 & 3.0 & 1.0 \\
\hline GCA & $6.5 \pm 0.1$ & & $1.0 \pm 0.1$ & & $2.4 \pm 0.4$ & 6 & 7.8 & 0.1 \\
\hline GCDCA & $1.6 \pm 0.1$ & & $1.3 \pm 0.1$ & & $2.9 \pm 0.1$ & 18 & 2.7 & 0.5 \\
\hline GDCA/GCA & $2.7 \pm 0.1$ & 2.7 & $1.9 \pm 0.1$ & 2 & $2.4 \pm 0.2$ & & & \\
\hline GDCA/GCDCA & $1.8 \pm 0.1$ & 1.6 & $1.6 \pm 0.2$ & 2.2 & $2.4 \pm 0.2$ & & & \\
\hline GCA/GCDCA & $2.7 \pm 0.2$ & 2.6 & $1.4 \pm 0.1$ & 1.2 & $2.3 \pm 0.1$ & & & \\
\hline GCDCA/GCA/GDCA & $1.9 \pm 0.2$ & 2.3 & $1.8 \pm 0.2$ & 1.6 & $2.4 \pm 0.2$ & & & \\
\hline
\end{tabular}

${ }^{a}$ The predicted values for CSI and CMC are also shown assuming that the mixtures of BS behave as an ideal solution. The values of aggregation number obtained by others for micelles in similar conditions to our studies are also given. ${ }^{b}$ The CMC of the mixtures is calculated from molar fraction of each component in solution $\left(x_{i}\right)$ and its CMC when pure $\left(\mathrm{CMC}_{i}\right), \frac{1}{\mathrm{CMC}}=\sum \frac{X_{i}}{\mathrm{CMC}_{i}} \cdot{ }^{47}{ }^{c} \mathrm{CSI}$ predicted for the complex BS mixtures using the average value of cholesterol solubilization obtained for each single BS weighted by their molar fraction. ${ }^{d}$ Aggregation number per micelle; values obtained from the literature. ${ }^{17,23,24}$ 
294 The same values for CSI were obtained from both methods, 295 showing that the chemical shift of BS resonances is a reliable 296 method to quantify solubilization of cholesterol even for more 297 complex mixtures (binary and ternary) of BS. GDCA, which 298 was the BS solubilizing more cholesterol, is the one with lowest 299 water solubility (lowest CMC) and the highest hydrophobicity. 300 The most hydrophilic BS (GCA) showed the lowest 301 solubilizing capacity for cholesterol. Surprisingly, although 302 GCDCA has a similar CMC to GDCA, its cholesterol 303 solubilizing capacity is closer to that of GCA. Similar 304 qualitative, but not quantitative, results were previously 305 obtained by other authors for the same BS. ${ }^{10,15,23}$ This 306 emphasizes that the change in the hydroxyl position between 307 the structures of GDCA and GCDCA does not affect their 308 solubility in water as a monomer but considerably modifies the 309 capacity of the hydrophobic core of the micelles to 310 accommodate cholesterol. The studied BS mixtures showed a 311 CSI equal to the one obtained using the average of CSI of each 312 BS weighted by their molar fraction. An exception is observed 313 for the GDCA/GCDCA mixture, which showed a lower 314 capacity to solubilize cholesterol than that expected from the 315 average of each BS. The capacity of the mixture GCA/GCDA 316 to solubilize cholesterol is also slightly higher than predicted 317 from the average of the CSI for the single component BS 318 micelles, although this is in agreement with the strong decrease 319 observed in the CMC of the mixture. The significant decrease 320 in the CSI observed for the GDCA/GCDCA mixture is not in 321 concordance with the effects observed on the CMC (only 322 slightly larger than predicted from ideal mixing). This suggests 323 an increased perturbation of the micelles' core and reinforces 324 the conclusion that the presence of the hydroxyl group (see 325 structure of BS at Figure 1) at C7 has a particularly high 326 destabilizing effect regarding cholesterol solubility.

327 As expected from the results obtained with single component 328 micelles, GCA/GCDCA mixtures show the lower cholesterol 329 solubilizing capacity. The addition of GDCA to this mixture 330 decreases the CMC and increases cholesterol solubility. The 331 surprising result obtained from this work is that the addition of 332 GCDCA to GDCA leads almost to the same effectivity in 333 reducing the cholesterol solubilizing capacity. To the best of 334 our knowledge, this is the first time that this effect among 335 different BS compositions has been evaluated.

336 To gain insight regarding changes in the structure of the 337 mixed micelles, their size has been measured using DLS. For 338 single-component micelles, the size obtained correlates well 339 with the micelle size and aggregation number obtained from 340 literature (Table 1), with smaller micelles for the three hydroxyl 341 BS and micelles from DCA derivatives being slightly smaller 342 than for CDCA. ${ }^{48}$ The results obtained in this work for the 343 micelles in the binary and ternary BS mixtures clearly show that 344 the accommodation of the distinct BS in the same micelle leads 345 to smaller micelles with a consequent increase in the 346 concentration of micelles for the same total BS concentration. 347 The results obtained for the biologically relevant ternary 348 mixture shows that the system is not optimized for CSI (neither 349 maximal nor minimal). It does suggest a preference for a large 350 quantity of very small micelles maintaining a relatively high 351 CSI.

352 To better compare the results of cholesterol solubilization 353 obtained by us with the results of others groups, we will use the 354 ratio of the concentration of cholesterol solubilized in the 355 micelles to the concentration of micelles $\left([\mathrm{Chol}]_{\mathrm{M}} /[\right.$ Micelles $\left.]\right)$.
The aggregation number used for GDCA, GCA, and GCDCA 356 micelles is shown in Table 1.

These results allow us to calculate the quantity of sterol per 358 micelle (mean occupation number), which are also given in 359 Table 1 for the simple BS micelles. The results obtained show 360 that GDCA micelles contain an average of one molecule of 361 cholesterol per micelle, these micelles being those that best 362 accommodate cholesterol. The other dihydroxy BS, GCDCA, 363 shows half of the solubilizing capacity of GDCA. GCA shows 364 even less affinity for cholesterol with a mean occupancy of 0.1. 365 The results are consistent with the available literature on 366 cholesterol solubilization, where GDCA shows an average 367 cholesterol solubilization per micelle of 0.9, GCDCA 0.4, and 368 GCA 0.2. ${ }^{10,15,23}$

369

The solubilization profiles obtained show an essentially 370 complete solubilization of cholesterol up to the saturation limit 371 for all BS and BS mixtures tested (Figure 3). This, together 372 with the small mean occupancy numbers, clearly indicates that 373 the association between cholesterol and the BS micelles cannot 374 be treated as binding to well-defined sites but rater by a 375 partition between the aqueous intermicellar space and the 376 micellar pseudophase. ${ }^{49}$ At the CSI, the BS aqueous solution is 377 saturated with cholesterol. Whether saturation of the solution is 378 due to the achievement of the limiting solubility in the aqueous 379 or micellar phases cannot be judged easily. At a given 380 concentration of total cholesterol, the concentration in the 381 aqueous and micellar phases depends on the relative affinity for 382 the two phases (partition coefficient). If the partition coefficient 383 was known, the concentrations in each phase could be 384 calculated and the comparison between the values obtained at 385 the CSI and those at saturation when each single phase is 386 present would lead to the unequivocal attribution of saturation 387 in the aqueous or micellar phase. This is not possible, however, 388 due to the low solubility of cholesterol in the aqueous phase 389 and the impossibility of have micelles as a single phase solution. 390 This discussion is nevertheless relevant because saturation of 391 the micellar pseudophase could compromise the solubilization 392 of other nonpolar solutes and reduce their intestinal absorption. 393 The observation that the maximal solubility of nonpolar 394 compounds (not prone to form micelles by their one) in 395 micellar solutions decreases as their hydrophobicity increases, ${ }^{49} 396$ is strong evidence for solubility being limited by saturation of 397 the aqueous phase.

398

The values obtained for the CSI in the distinct micellar 399 solutions, Table 1, show that the affinity of cholesterol for the 400 single component BS micelles is highest for GDCA and lowest 401 for GCA. The affinity of cholesterol for the physiologically 402 relevant ternary mixture of BS is intermediate, showing that the 403 system is not optimized for a maximal cholesterol solubility. In 404 addition to the eventual preference for smaller size micelles (as 405 discussed above), the physiological composition of BS micelles 406 in the intestinal lumen may reflect an additional step required 407 in the process of cholesterol absorption-association with the 408 apical membrane of the brush border cells in the intestinal 409 epithelium. To be absorbed the solutes must associate with the 410 membranes of the epithelium (either with the lipid bilayer for 411 passive absorption or with specific transporters in the 412 membrane). This association is not mediated by dissolution 413 of the BS micelles with the membrane, but rather it is mediated 414 by the solute in the aqueous phase. Therefore, maximal 415 absorption is a trade-off between efficient solubilization in the 416 intestinal lumen and a relatively large concentration in the 417 
418 aqueous phase to allow partition to the gastrointestinal 419 membrane.

420 Effect of Phytosterols on Cholesterol Solubilization in 421 GDCA Micelles. Several compounds are known to affect 422 cholesterol absorption in the intestine and have been implicated 423 in changes in the solubilization capacity of the dietary mixed 424 micelles (DMM) in the intestinal lumen. Phytosterols are 425 probably one of the most studied families of molecules for this 426 purpose. $^{23,41,50}$ Fatty acids (FA), namely the unsaturated ones, 427 and some vitamins (namely $\alpha$-tocopherol) are also involved in 428 the decrease of cholesterol absorption and other lipophilic 429 molecules. ${ }^{51}$ To understand the mechanism of action of these 430 compounds and, most importantly, to quantitatively determine 431 their effect, we used our previously established NMR method ${ }^{19}$ 432 to follow the solubilization of both cholesterol and dietary 433 compound in the BS micelles. Figure 4 shows an expansion of a

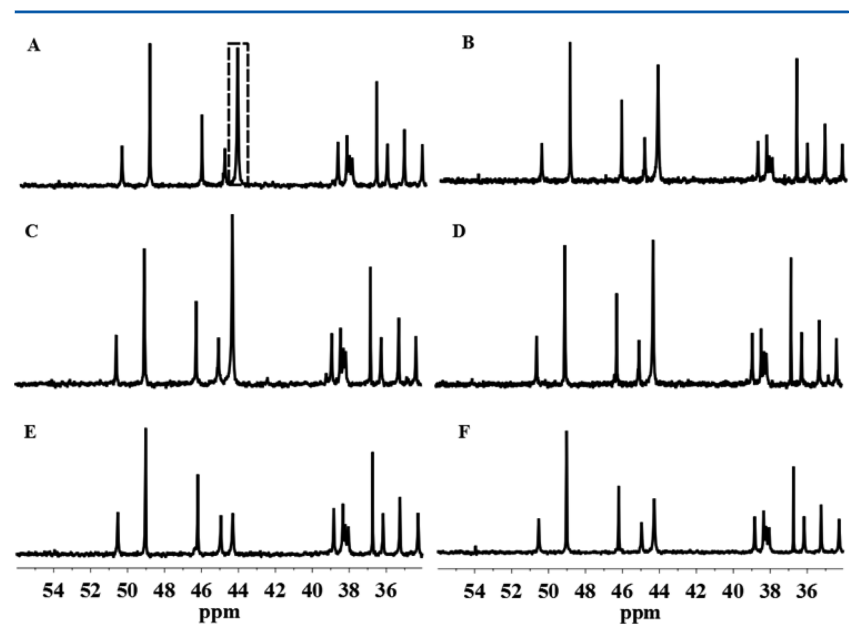

Figure 4. ${ }^{13} \mathrm{C}$ NMR expanded spectra of $50 \mathrm{mM}$ GDCA with $3.5 \mathrm{mM}$ $\left[4-{ }^{13} \mathrm{C}\right]$ cholesterol (A) and in the presence of $3.5 \mathrm{mM}$ of dietary phytochemicals: $\alpha$-tocopherol (B), palmitic acid (C), oleic acid (D), stigmastanol (E), and stigmasterol (F). The spectra were acquired with ${ }^{1} \mathrm{H}$ decoupling and NOE, in $\mathrm{D}_{2} \mathrm{O}$ aqueous buffer at $37^{\circ} \mathrm{C}$. The peak of ${ }^{13} \mathrm{C}_{4}$ enriched cholesterol appears at $44 \mathrm{ppm}$ (black dotted box). Note the relative height of the peak at $44 \mathrm{ppm}$ (cholesterol) showing an increase in the presence of palmitic acid and a decrease in the presence of all other phytochemicals.

434 typical ${ }^{13} \mathrm{C}$ NMR spectrum of GDCA micelles acquired in the 435 presence of labeled $\left[4-{ }^{13} \mathrm{C}\right]$ cholesterol and of various dietary 436 phytochemicals. The resonance at $44 \mathrm{ppm}$, corresponding to 437 carbon four $\left(\mathrm{C}_{4}\right)$ of labeled $\left[4-{ }^{13} \mathrm{C}\right]$ cholesterol, allows the 438 quantitative determination of cholesterol solubilized in the 439 micelles.

440 The results clearly showed that stigmasterol and stigmastanol 441 were most effective at reducing cholesterol dissolution by BS 442 micelles. They belong to two classes of phytosterols designated 443 as sterols and stanols, respectively, known as inhibitors of 444 cholesterol absorption. Two mechanisms can explain the 445 decrease of cholesterol solubility in the presence of 446 phytosterols. $^{26,37,50}$ One possibility is the competition of both 447 molecules for solubilization in the hydrophobic core of BS 448 micelles in a process referred to as cosolubilization. 449 Alternatively, the decrease in cholesterol solubilization may 450 be due to a reduction of its aqueous solubility due to the 451 formation of mixed crystals with the phytosterols, a mechanism 452 of coprecipitation. The two processes are not mutually 453 exclusive and therefore a combination of both processes may occur. The distinction between the two mechanisms is of 454 practical relevance because if the coprecipitation is the major 455 one, the capacity of BS micelles to solubilize other nonpolar 456 solutes is expected to be unaffected. Otherwise, the use of high 457 concentrations of phytosterols in the diet could lead to a 458 deficient absorption of other important nonpolar solutes such 459 as vitamins and/or pharmaceuticals.

Insight regarding the mechanism of the decrease in 461 cholesterol solubilization may be obtained from the simulta- 462 neous quantification of cholesterol and dietary food ingredients 463 solubilized by the BS micelles. Stigmasterol structure is very 464 similar to that of cholesterol except for an additional double 465 bond in the side chain (see Figure 1). This allowed the direct 466 quantification of both sterols by NMR. The proton spectrum of 467 cholesterol in BS micelles (Figure 5) has a peak at 5.3 ppm, $468 \mathrm{fs}$

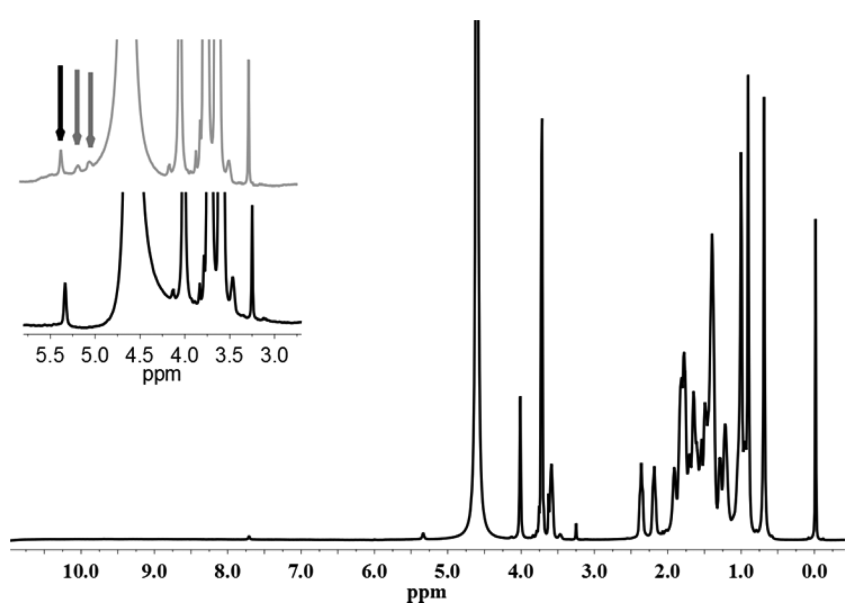

Figure 5. ${ }^{1} \mathrm{H}$ NMR spectra of $50 \mathrm{mM}$ GDCA micelles in $\mathrm{D}_{2} \mathrm{O}$ buffer Tris-HCL pH $=7.4$ with $3.5 \mathrm{mM}\left[4-{ }^{13} \mathrm{C}\right]$ cholesterol. The inset show an expansion from 5.5 to $3.0 \mathrm{ppm}$ with the peak of cholesterol solubilized in micelles (black line) and the peaks from cholesterol and stigmasterol (gray line). The arrows indicate the resonance from cholesterol (black) and from stigmasterol (gray) additional two alkene protons.

which is characteristic of alkene protons. Stigmasterol also has a 469 proton at the $\mathrm{C} 4$ position that resonates at this same frequency 470 (black arrow in Figure 5). The two additional protons of the 471 double bond in the side chain resonate closer to the water 472 frequency at $4.6 \mathrm{ppm}$ (gray arrows in Figure 5). Although these 473 two latter signals could not be used to quantify stigmasterol due 474 to baseline distortions caused by the proximity to the water 475 resonance, the $5.3 \mathrm{ppm}$ signal of the protons located in the 476 cholesterol and stigmasterol backbone could be informative of 477 the solubilization of both sterols.

478

While in the proton spectrum the area of the signal at 5.3479 ppm corresponds to both sterols solubilized in the micelle, in 480 the ${ }^{13} \mathrm{C}$ NMR spectrum, only cholesterol is quantified because 481 only it, and not stigmasterol, is enriched in ${ }^{13} \mathrm{C}$. Thus, using a 482 known quantity of TSP as the internal reference, the total 483 concentration of solubilized sterols was quantitatively deter- 484 mined from the $5.3 \mathrm{ppm}$ proton peak area. The concentration 485 of cholesterol in the same solution was obtained from 486 quantitative ${ }^{13} \mathrm{C}$ NMR spectra. ${ }^{19}$ Therefore, the difference 487 between both concentrations gave the solubility of stigmasterol 488 in GDCA micelles. Figure 6 shows the results obtained for the $489 \mathrm{f} 6$ effect of stigmasterol on cholesterol solubility in GDCA 490 




[Stigmasterol] $(\mathrm{mM})$

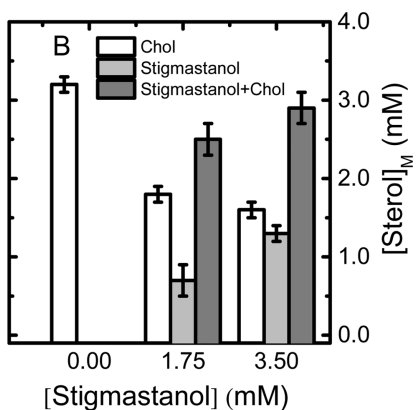

[Stigmastanol] $(\mathrm{mM})$
Figure 6. Effect of stigmasterol (A) and stigmastanol (B) on cholesterol $(3.5 \mathrm{mM})$ solubilization by $50 \mathrm{mM}$ GDCA micelles. The average and standard errors associated with values of solubilization at equilibrium for both cholesterol and stigmasterol in the GDCA micelles $\left([\text { Sterol }]_{M}\right)$ obtained are from at least three independent experiments.

491 micelles, as well as the solubility of stigmasterol and the total 492 sterol concentration.

493 In the absence of dietary phytochemicals, the solubility of 494 cholesterol in a $50 \mathrm{mM}$ GDCA solution is $3.2 \mathrm{mM}$. This value 495 is higher than the CSI reported in Table 1 for GDCA micelles 496 because in the competition study with cosolutes, a $50 \mathrm{mM}$ total 497 concentration of BS was used, while in the previous 498 measurements the total concentration of cholesterol plus BS 499 was $50 \mathrm{mM}$.

500 As expected, the amount of cholesterol solubilized by the BS 501 micelles decreases in the presence stigmasterol. This phytoster$502 \mathrm{ol}$ is also solubilized by GDCA micelles, although the total 503 amount of sterols solubilized (cholesterol + stigmasterol) 504 decreases. The fraction of each sterol solubilized by the GDCA 505 micelles is essentially equal to the total fraction in solution 506 suggesting no preferential solubilization of any of the sterols. 507 To the best of our knowledge, this is the first time that 508 solubilized cholesterol and phytosterol are measured directly in 509 the same micellar solution using noninvasive techniques. 510 Previous reports required the physical separation between 511 micelles and precipitates, such as filtration or chromatography, 512 and significant perturbations in the equilibrium could occur. 513 The observation that the total sterol solubilized decreases as the 514 proportion of stigmasterol is increased points toward the 515 involvement of coprecipitation with a lower aqueous solubility 516 for the mixture cholesterol plus stigmasterol than that of 517 cholesterol alone. Sterol solubility in aqueous solutions is not 518 known with high accuracy, but available data in the literature 519 indicates a smaller solubility of stigmasterol as compared with cholesterol. ${ }^{52}$ This is in fact expected due to the additional two 520 carbons in the side chain, which are not compensated by the 521 extra double bond. Another evidence for coprecipitation was 522 obtained from the analysis of formed crystals by polarized 523 microscopy (data not shown), which show crystal morpholo- 524 gies different from those of cholesterol alone, and are in 525 agreement with data shown in the literature. ${ }^{50} 526$

The solubilization of cholesterol and stigmastanol in GDCA 527 micelles was also characterized and is shown in Figure 6, panel 528 B. As observed for stigmasterol, the presence of stigmastanol 529 leads to a decrease in cholesterol solubility. However, the 530 results suggest a relatively higher effect at a cholesterol:- 531 stigmastanol ratio of 0.5 .

To elucidate the mechanism involved, it would be important 533 to quantify the solubility of stigmastanol in GDCA micelles. 534 The method used above for stigmasterol cannot be used due to 535 the absence of a specific proton or carbon resonance from 536 stigmastanol that could be separately observed and used for the 537 quantification of its micellar solubilization. The quantification 538 of stigmastanol in the GDCA micelles was quantitatively 539 followed through its effects in the resonances of GDCA, using 540 quantitative chemical shift analysis (qCSA). ${ }^{19}$ This method- 541 ology may be used to quantify the total sterol solubilized in the 542 micelles, considering that the effect induced by the solutes is 543 known. Due to their similar chemical structures, this effect is 544 expected to be the same for both cholesterol and the 545 phytosterols. This approach was first validated using 546 stigmasterol by comparison with the results obtained from its 547 direct quantification by ${ }^{1} \mathrm{H}$ NMR (see above). Table S3 in the 548 Supporting Information shows the chemical shift variation and 549 the slopes of such plots obtained for the micelles in the 550 presence of cholesterol or cholesterol plus stigmasterol. The 551 slopes relating chemical shift with solubilized sterol show 552 similar values in the two situations. This allows determination 553 of the total solubilized sterol by following the chemical shift of 554 the most sensitive BS resonances. The determined value of 555 solubility was equal to the value of solubilized sterol in the 556 micelle determined by quantitative $1 \mathrm{H} \mathrm{NMR}$ (Table 2). This $557 \mathrm{t} 2$ demonstrates the robustness of the method and the validity of 558 the initial assumption regarding similar locations and effects in 559 the BS micelles. This same strategy was followed for 560 stigmastanol, allowing the quantification of stigmastanol 561 solubility in the GDCA micelle in the presence of cholesterol 562 (Table S4 of the Supporting Information).

The results obtained for the amount of stigmastanol and total 564 sterol solubilized in the GDCA micelles is shown in Figure 6B 565

Table 2. Comparison between Total Sterol (Cholesterol + Stigmasterol) Solubilized in GDCA Micelles Determined by Quantitative ${ }^{13} \mathrm{C}$ and ${ }^{1} \mathrm{H}$ NMR Spectroscopy and the Total Sterol Determined from Quantitative Chemical Shift Analysis $(\mathrm{qCSA})^{a}$

\begin{tabular}{|c|c|c|c|c|c|c|}
\hline & $\mathrm{mM}$ & cholesterol & chol/stigmasterol 2:1 & chol/stigmasterol 1:1 & chol/stigmastanol 2:1 & chol/stigmastanol 1:1 \\
\hline \multirow[t]{3}{*}{ quantitative NMR } & {$[\mathrm{Chol}]_{\mathrm{M}}$} & $3.2 \pm 0.1$ & $1.6 \pm 0.2$ & $1.0 \pm 0.2$ & $1.8 \pm 0.1$ & $1.6 \pm 0.1$ \\
\hline & {$[\text { Stigmasterol }]_{\mathrm{M}}$} & & $0.6 \pm 0.2$ & $1.0 \pm 0.1$ & & \\
\hline & {$[\text { Total Sterol }]_{M}$} & & $2.2 \pm 0.3$ & $2.0 \pm 0.1$ & & \\
\hline \multirow[t]{3}{*}{ qCSA NMR } & {$[\text { Total Sterol }]_{\mathrm{M}}$} & & $2.1 \pm 0.3$ & $2.0 \pm 0.1$ & $2.5 \pm 0.2$ & $2.9 \pm 0.2$ \\
\hline & {$[\text { Stigmastanol }]_{M}{ }^{b}$} & & & & $0.7 \pm 0.2$ & $1.3 \pm 0.1$ \\
\hline & {$[\text { Stigmasterol }]_{\mathrm{M}}^{b}$} & & $0.5 \pm 0.1$ & $1.0 \pm 0.1$ & & \\
\hline
\end{tabular}

${ }^{a}$ The determination of stigmastanol solubilized in the micelle was done using the same chemical shift analysis as for stigmasterol. The total cholesterol present in the solutions was $3.5 \mathrm{mM}$, and the concentration of GDCA was always $50 \mathrm{mM}$. ${ }^{b}$ The concentration of stigmasterol and stigmastanol was obtained by the difference between the total sterol obtained by chemical shift analysis and the concentration of cholesterol determined by ${ }^{13} \mathrm{C}$ NMR quantification for at least three independent experiments. 
566 and Table 2. The absence of double bonds in stigmastanol 567 would predictably decrease its solubility in aqueous solutions, 568 even below that of stigmasterol. Data available in the literature, 569 however, indicates otherwise, with the solubility of stigmastanol 570 being between that of cholesterol and stigmasterol. ${ }^{52}$ The 571 observation that the total amount of sterol solubilized in the 572 GDCA micelles does not change significantly as the 573 concentration of stigmastanol is increased suggests that the 574 overall sterol solubility is not significantly affected.

575 The literature regarding solubilization of phytosterols and 576 their quantitative effects on decreasing cholesterol solubility in 577 BS micelles is not consensual. Cholesterol and stigmasterol 578 solubility in nonconjugated BS was studied as a function of BS 579 concentration. The results showed that the presence of 580 stigmasterol in deoxycholate micelles, at a similar concentration 581 to the one used in our work, induced a mild decreasing effect 582 on cholesterol solubility. ${ }^{27}$ In the same work, cholestanol (a 583 stanol similar to stigmastanol) showed a strong decrease in the 584 amount of solubilized cholesterol. Also, the total sterol 585 solubilized at the BS micelle as compared with the cholesterol 586 solubilized in the absence of phytosterols was lower for the case 587 of cholestanol and similar for the case of stigmasterol. Other 588 authors have encountered a significant increase in the total 589 amount of sterol solubilized by taurine-conjugated DCA when 590 both cholesterol and sitosterol or sitostanol are present (the 591 amount of cholesterol solubilized being reduced to similar 592 extent by both phytosterols). ${ }^{52}$ The same authors have 593 characterized the effects of several phytosterols, and the results 594 encountered were most frequently an increase in the total 595 amount of sterol solubilized in the BS micelles despite the 596 smaller solubility of the phytosterols alone and a reduction in 597 the amount of cholesterol solubilized in the presence of the 598 phytosterol. $^{40}$ Those results are unexpected, both when 599 interpreted as competition for solubilization in the BS micelle 600 or coprecipitation in mixed crystals. The methodologies used to 601 quantify the amount of sterols solubilized in the micelles, 602 involving the physical separation between the micelles, may be 603 the basis for those discrepancies. In fact, with the approach 604 followed in previous work, it is not possible to distinguish 605 between sterol in small crystals (smaller than $0.2 \mu \mathrm{m}$ ) and 606 solubilized in BS micelles. Another source of discrepancy may 607 be due to different methodologies followed in the sterol 608 solubilization by the BS micelles. The distinct sterols were 609 added to the BS solution as a mixture of preformed single 610 crystals. In this work, a film of the distinct sterols was prepared 611 by solvent evaporation from a homogeneous solution, thus 612 favoring the formation of mixed crystals when the aqueous 613 solution containing the BS is added. This methodology is 614 physiologically more relevant, as sterol precipitation is expected 615 to occur during digestion, as the emulsifying lipids from diet are 616 progressively digested.

617 Effect of Saturated and Unsaturated Fatty Acids on ${ }_{618}$ Cholesterol Solubilization in GDCA Micelles. To study the 619 effect of saturated and unsaturated FA on cholesterol 620 solubilization, we used palmitic (PA) and oleic acid (OA). 621 Those two fatty acids were chosen because they have a similar 622 solubility in aqueous solutions. ${ }^{53}$ Therefore, the effect on 623 cholesterol solubility should be due only to the presence of the 624 cis double bond (and not also due to a different aqueous 625 solubility as would be the case for oleic and stearic acid). The 626 results obtained are shown in Figure 7.

627 The presence of the saturated palmitic acid leads to a small 628 increase in the solubility of cholesterol. For these experiments,


Figure 7. Effect of saturated (palmitic acid - left panel) and unsaturated fatty acids (oleic acid - right panel) on cholesterol solubilization. Values shown are for fatty acids/cholesterol ratios equal to $0,0.5$, and 1 . BS composition is $50 \mathrm{mM}$ GDCA, and the cholesterol is $3.5 \mathrm{mM}$ in all experiments. The values indicated for the solubility are the average and standard deviation of at least three independent experiments. The solubility of the FA in the BS micelles in the presence of cholesterol is also shown being obtained by ${ }^{1} \mathrm{H}$ NMR (for $\mathrm{OA}$ ) or by qCSA (for PA).

two different assays were performed. In the first one, a film of 629 cholesterol and FA was prepared and allowed to equilibrate 630 with the BS aqueous solution for $24 \mathrm{~h}$. In the other assay, a pre- 631 equilibrated solution of BS with FA was added to the 632 cholesterol film. The results obtained from the two experiments 633 were similar and are included in the results shown in Figure 7.634 In the absence of FA, GDCA micelles solubilize $3.2 \mathrm{mM}$ out of 635 a total cholesterol concentration of $3.5 \mathrm{mM}$. Addition of 1.75636 mM PA to the BS micelles leads to a slight increase of 637 cholesterol solubility. Doubling the concentration of PA in the 638 system leads to the total solubilization of the cholesterol 639 present in solution. A higher cholesterol concentration (5 mM) 640 was used for both PA concentrations (1.75 and $3.5 \mathrm{mM}$ ), but 641 no further solubilization was achieved (results not shown). The 642 results showed that the presence of PA in the BS micelles leads 643 to a modest increase on the solubility of cholesterol in small 644 micelles.

645

The analysis of the changes in the ${ }^{13} \mathrm{C}$ chemical shifts of the 646 different resonances of BS upon cholesterol and FA addition 647 showed that several resonances were sensitive to the increase of 648 FA in the system and could be used to quantify solubilized FA. 649 Table S5 (Supporting Information) shows a typical example of 650 the quantitative determination of the solubilized PA in the BS 651 micelles followed by qCSA. This result demonstrates that PA 652 was completely solubilized by BS micelles at both concen- 653 trations used.

The results obtained for cholesterol solubility in the presence 655 of OA (Figure 7, right panel) showed a different trend when 656 compared with PA. The unsaturated OA leads to a slight 657 decrease in cholesterol solubility, which is very small when 658 compared with the effect of phytosterols in the same 659 proportions. The solubility of OA in the micelles was also 660 followed by quantitative ${ }^{1} \mathrm{H}$ NMR and by chemical shift 661 analysis (Table S6, Supporting Information). Complete 662 solubilization of OA was obtained, at both concentrations 663 used $(1.75$ and $3.5 \mathrm{mM})$. This result is different from that 664 observed for phytosterols, where the dietary compound was not 665 completely solubilized by the micelles and showed a defined 666 solubility limit. This was consistent with observations done 667 with DMM where oleic acid was shown to decrease cholesterol 668 solubility when high OA/cholesterol ratios were used. ${ }^{41} \quad 669$ 
670 Evidence for the solubilization of both FA and cholesterol in 671 the BS micelles was obtained from the chemical shift variations 672 of BS induced by the presence of the solutes and by the absence 673 of large FA micelles as measured by DLS. No significant 674 increase in the size of the mixed micelles was observed, as 675 expected from the small number of solute molecules solubilized 676 in each BS micelle.

677 Effect of $\boldsymbol{\alpha}$-Tocopherol on Cholesterol Solubilization 678 in GDCA Micelles. Vitamin E is one of the most abundant 679 lipid-soluble antioxidants in higher mammals. ${ }^{51}$ It can exist in 680 two forms: tocopherols and tocotrienols. $\alpha$-Tocopherol 681 (molecular structure in Figure 1) is one of the most 682 representative forms of tocopherols. Its effect on cholesterol 683 solubilization by BS micelles was studied, and the results 684 obtained are shown in Figure 8.

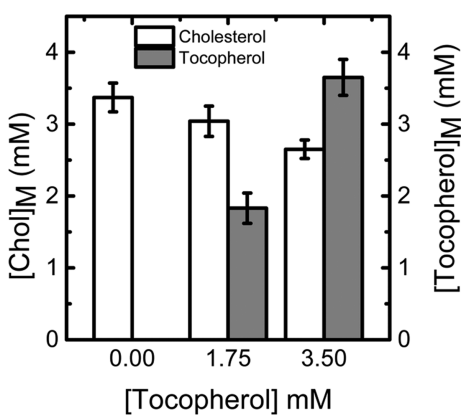

Figure 8. Effect of tocopherol on cholesterol solubility by the BS micelles. The values shown are for tocopherol/cholesterol ratios of 0 , 0.5 , and 1. The BS composition is $50 \mathrm{mM}$ GDCA and the total cholesterol content used in each experiment is $3.5 \mathrm{mM}$. The solubility values are the average and standard deviation of at least three independent experiments. The solubilization of tocopherol in the BS micelles in the presence of sterol was measured by UV absorption spectroscopy, and qCSA and is also shown.

685 The solubilization profile shows a decrease of cholesterol 686 solubility in the presence of $\alpha$-tocopherol. This effect is clearly 687 lower than that of phytosterols, but is higher than that observed 688 for oleic acid. Tocopherol solubility in GDCA micelles was 689 followed by UV absorption spectroscopy and by qCSA (Table 690 S7, Supporting Information), and it was complete for both 691 concentrations studied.

\section{CONCLUSIONS}

693 The results presented in this work clearly show that the BS 694 micelles composition present in vivo is not optimized for the 695 highest solubilizing capacity, instead they show a compromise 696 between the concentration of micelles, their size, and the ability 697 to solubilize cholesterol. This reflects the dual physiological role 698 of BS micelles in the intestinal lumen, which not only help in 699 the solubilization of hydrophobic molecules but also work as a 700 vehicle for their delivery to the intestinal membrane.

701 In order to understand the effect of diet changes on 702 cholesterol solubility by BS micelles in the intestinal lumen, we 703 studied the effect of several hydrophobic molecules, such as 704 phytosterols (stigmasterol and stigmastanol), fatty acids 705 (saturated palmitic acid and monounsaturated oleic acid) and 706 vitamin E ( $\alpha$-tocopherol). These molecules, namely, phytoster707 ols, unsaturated FA, and tocopherol, are known to decrease 708 cholesterol absorption in the intestine, ${ }^{1,54}$ although the 709 mechanism is not fully understood. ${ }^{22,26,37,38,41}$ The method710 ology used in this work permits the direct quantification of both cholesterol and dietary food ingredient, allowing in this way a 711 better elucidation of the mechanism involved in the effect on 712 cholesterol solubilization.

713

The results obtained show that all dietary food ingredients 714 tested are cosolubilized with cholesterol in the BS micelles. 715 This cosolubilization leads to a decrease in the maximal 716 solubility of cholesterol for most dietary food ingredients tested 717 (phytosterols, $\alpha$-tocopherol, and OA) and to an increase for the 718 case of the saturated fatty acid (PA). The total amount of solute 719 solubilized by the BS micelles (cholesterol plus dietary food 720 ingredient) decreases in the case of phytosterols (more 721 pronounced for stigmasterol) and increases for all other food 722 ingredients added with its complete solubilization in the BS 723 micelles. Those results clearly show that saturation of the BS 724 micelles capacity to accommodate solutes is not the mechanism 725 involved in the reduction of cholesterol solubilization by the BS 726 micelles. By contrast, effects in the maximal cholesterol 727 solubility in the micellar solution should reflect variations in 728 its tendency to precipitate (coprecipitation with the added 729 dietary food ingredient, as observed in the case of the 730 phytosterols) and/or variations in the properties of the mixed 731 micelles formed by the BS and the added food ingredient (as 732 observed for the case of FA and $\alpha$-tocopherol). In the former 733 mechanism, the efficiency of the decrease in cholesterol 734 solubilization depends on the aqueous solubility of the dietary 735 food ingredient added and on the relative stability of mixed 736 crystals. The efficiency observed in the later mechanism 737 depends on the relative affinity of cholesterol for BS micelles 738 when pure and mixed with the dietary food ingredient. In this 739 respect, the shape and rigidity of the added food ingredient 740 plays a crucial role due to the interactions with the rigid steroid 741 moiety of cholesterol. The understanding of those mechanisms 742 and the molecular constraints involved is the first step toward 743 the design of better strategies to decrease cholesterol 744 absorption and/or in the optimization of BS-based models 745 for drug delivery to the gastrointestinal membrane.

\section{ASSOCIATED CONTENT}

\section{S Supporting Information}

748

The Supporting Information is available free of charge on the 749 ACS Publications website at DOI: 10.1021/acs.lang- 750 muir.6b00723.

Additional tables and details as described in the text 752 (PDF)

\section{AUTHOR INFORMATION}

Notes

The authors declare no competing financial interest.

\section{ACKNOWLEDGMENTS}

The Coimbra Chemistry Centre (CQC) is supported by the 758 Portuguese "Fundação para a Ciência e a Tecnologia" (FCT) 759 through the Project No. 007630 UID/QUI/00313/2013, 760 cofunded by COMPETE2020-UE. This work was also 761 supported by project RECI/QEQ-QFI/0168/2012. F.M.C.-G. 762 acknowledges FCT for the Ph.D. fellowship SFRH/BD/40778/ 763 2007. We acknowledge the Rede Nacional de RMN for access 764 to the facilities. The Varian VNMRS $600 \mathrm{MHz}$ spectrometer is 765 part of the National NMR Network (PTNMR) and was 766 purchased in the framework of the National Program for 767 Scientific Re-equipment, contract REDE/1517/RMN/2005, 768 with funds from POCI 2010 (FEDER) and FCT. We also 769 
770 acknowledge the Centre for Neuroscience and Cell Biology for 771 access to the DLS.

\section{$772 \square$ REFERENCES}

773 (1) Wilson, M. D.; Rudel, L. L. Review of Cholesterol Absorption 774 with Emphasis on Dietary and Biliary Cholesterol. J. Lipid Res. 1994, 77535 (6), 943-955.

776 (2) Glass, C. K.; Witztum, J. L. Atherosclerosis: The road ahead. Cell 777 2001, 104 (4), 503-516.

778 (3) Haberlan, M. E.; Reynolds, J. A. Self-Association of Cholesterol in 779 Aqueous-Solution. Proc. Natl. Acad. Sci. U. S. A. 1973, 70 (8), 23137802316.

781 (4) Staggers, J. E.; Hernell, O.; Stafford, R. J.; Carey, M. C. Physical782 Chemical Behavior of Dietary and Biliary Lipids During Intestinal 783 Digestion and Absorption 0.1. Phase-Behavior and Aggregation States 784 of Model Lipid Systems Patterned After Aqueous Duodenal Contents 785 of Healthy Adult Human-Beings. Biochemistry 1990, 29 (8), 20287862040.

787 (5) Watt, S. M.; Simmonds, W. J. Specificity of Bile-Salts in 788 Intestinal-Absorption of Micellar Cholesterol in Rats. Clin. Exp. 789 Pharmacol. Physiol. 1976, 3 (4), 305-322.

790 (6) Westergaard, H.; Dietschy, J. M. Mechanism Whereby Bile-Acid 791 Micelles Increase Rate of Fatty-Acid and Cholesterol Uptake Into 792 Intestinal Mucosal Cell. J. Clin. Invest. 1976, 58 (1), 97-108.

793 (7) Woollett, L. A.; Wang, Y.; Buckley, D. D.; Yao, L.; Chin, S.; 794 Granholm, N.; Jones, P. J. H.; Setchell, K. D. R.; Tso, P.; Heubi, J. E. 795 Micellar solubilisation of cholesterol is essential for absorption in 796 humans. Gut 2006, 55 (2), 197-204.

797 (8) Siperstein, M. D.; Chaikoff, I. L.; Reinhardt, W. O. C-14798 Cholesterol 0.5. Obligatory Function of Bile in Intestinal Absorption 799 of Cholesterol. J. Biol. Chem. 1952, 198 (1), 111-114.

800 (9) Carey, M. C.; Small, D. M. Physical-Chemistry of Cholesterol 801 Solubility in Bile - Relationship to Gallstone Formation and 802 Dissolution in Man. J. Clin. Invest. 1978, 61 (4), 998-1026.

803 (10) Igimi, H.; Carey, M. C. Cholesterol Gallstone Dissolution in 804 Bile - Dissolution Kinetics of Crystalline (Anhydrate and Mono805 hydrate) Cholesterol with Chenodeoxycholate, Ursodeoxycholate, and 806 Their Glycine and Taurine Conjugates. J. Lipid Res. 1981, 22 (2), 807 254-270.

808 (11) Wang, D. Q. H.; Cohen, D. E.; Carey, M. C. Biliary lipids and 809 cholesterol gallstone disease. J. Lipid Res. 2008, 50, S406-S411.

810 (12) Hofmann, A. F. Molecular Association in Fat Digestion. 811 Interaction in Bulk of Monoolein, Oleic Acid, and Sodium Oleate with 812 Dilute, Micellar Bile Salt Solutions. In Molecular Association in 813 Biological and Related Systems; Goddard, E. D., Ed.; American 814 Chemical Society Publications: Washington, DC, 1968; Vol. 84, pp 815 53-66.

816 (13) Donovan, J. M.; Timofeyeva, N.; Carey, M. C. Influence of 817 Total Lipid-Concentration, Bile-Salt Lecithin Ratio, and Cholesterol 818 Content on Inter-Mixed Micellar/Vesicular (Non-Lecithin-Associ819 ated) Bile-Salt Concentrations in Model Bile. J. Lipid Res. 1991, 32 820 (9), 1501-1512.

821 (14) Small, D. M.; Bourges, M.; Dervichian, Dg Ternary and 822 Quaternary Aqueous Systems Containing Bile Salt Lecithin and 823 Cholesterol. Nature 1966, 211 (5051), 816-818.

824 (15) Neiderhiser, D. H.; Roth, H. P. Cholesterol Solubilization by 825 Solutions of Bile Salts and Bile Salts Plus Lecithin. Exp. Biol. Med. 826 1968, 128 (1), 221-225.

827 (16) Armstrong, M. J.; Carey, M. C. Thermodynamic and Molecular 828 Determinants of Sterol Solubilities in Bile-Salt Micelles. J. Lipid Res. 829 1987, 28 (10), 1144-1155.

830 (17) Carey, M. C.; Montet, J. C.; Phillips, M. C.; Armstrong, M. J.; 831 Mazer, N. A. Thermodynamic and Molecular-Basis for Dissimilar 832 Cholesterol-Solubilizing Capacities by Micellar Solutions of Bile-Salts 833 Cases of Sodium Chenodeoxycholate and Sodium Ursodeoxycholate 834 and Their Glycine and Taurine Conjugates. Biochemistry 1981, 20 835 (12), 3637-3648.
(18) Carey, M. C.; Small, D. M. Micelle Formation by Bile-Salts - 836 Physical-Chemical and Thermodynamic Considerations. Arch. Intern. 837 Med. 1972, 130 (4), 506-527.

838

(19) Coreta-Gomes, F. M.; Vaz, W. L. C.; Wasielewski, E.; Geraldes, 839 C.; Moreno, M. J. Quantification of cholesterol solubilized in bile salt 840 micellar aqueous solutions using C-13 nuclear magnetic resonance. 841 Anal. Biochem. 2012, 427 (1), 41-48.

842

(20) Garidel, P.; Hildebrand, A.; Neubert, R.; Blume, A. 843 Thermodynamic characterization of bile salt aggregation as a function 844 of temperature and ionic strength using isothermal titration 845 calorimetry. Langmuir 2000, 16 (12), 5267-5275.

846

(21) Janich, M.; Lange, J.; Graener, H.; Neubert, R. Extended light 847 scattering investigations on dihydroxy bile salt micelles in low-salt 848 aqueous solutions. J. Phys. Chem. B 1998, 102 (31), 5957-5962. 849 (22) Jesch, E. D.; Carr, T. P. Sitosterol reduces micellar cholesterol 850 solubility in model bile. Nutr. Res. (N. Y., NY, U. S.) 2006, 26 (11), 851 $579-584$.

852

(23) Matsuoka, K.; Maeda, M.; Moroi, Y. Micelle formation of 853 sodium glyco- and taurocholates and sodium glyco- and taurodeox- 854 ycholates and solubilization of cholesterol into their micelles. Colloids 855 Surf., B 2003, 32 (2), 87-95.

(24) Matsuoka, K.; Suzuki, M.; Honda, C.; Endo, K.; Moroi, Y. 857 Micellization of conjugated chenodeoxy- and ursodeoxycholates and 858 solubilization of cholesterol into their micelles: comparison with other 859 four conjugated bile salts species. Chem. Phys. Lipids 2006, 139 (1), 1- 860 10.

(25) Mazer, N. A.; Carey, M. C. Quasi-Elastic Light-Scattering- 862 Studies of Aqueous Biliary Lipid Systems - Cholesterol Solubilization 863 and Precipitation in Model Bile Solutions. Biochemistry 1983, 22 (2), 864 426-442.

865

(26) Mel'nikov, S. M.; Seijen ten Hoorn, J. W. M.; Eijkelenboom, A. 866 P. A. M. Effect of phytosterols and phytostanols on the solubilization 867 of cholesterol by dietary mixed micelles: an in vitro study. Chem. Phys. 868 Lipids 2004, 127 (2), 121-141.

(27) Nagadome, S.; Okazaki, Y.; Lee, S.; Sasaki, Y.; Sugihara, G. 870 Selective solubilization of sterols by bile salt micelles in water: A 871 thermodynamic study. Langmuir 2001, 17 (14), 4405-4412. 872

(28) Neiderhiser, D. H.; Roth, H. P. Effect of Modifications of 873 Lecithin and Cholesterol on Micellar Solubilization of Cholesterol. 874 Biochim. Biophys. Acta, Lipids Lipid Metab. 1972, 270 (3), 407-413. 875

(29) Small, D. M. Size and Structure of Bile Salt Micelles. Influence 876 of Structure, Concentration, Counterion Concentration, $\mathrm{pH}$, and 877 Temperature. In Molecular Association in Biological and Related Systems; 878 Goddard, E. D., Ed.; American Chemical Society Publications: 879 Washington, DC, 1968; Vol. 84, pp 31-52.

(30) Coreta-Gomes, F. M.; Martins, P. A. T.; Velazquez-Campoy, A.; 881 Vaz, W. L. C.; Geraldes, C. F. G.; Moreno, M. J. Interaction of Bile 882 Salts with Model Membranes Mimicking the Gastrointestinal 883 Epithelium: A Study by Isothermal Titration Calorimetry. Langmuir 884 2015, 31 (33), 9097-9104.

885

(31) Chen, Y.; Lu, Y.; Chen, J.; Lai, J.; Sun, J.; Hu, F.; Wu, W. 886 Enhanced bioavailability of the poorly water-soluble drug fenofibrate 887 by using liposomes containing a bile salt. Int. J. Pharm. 2009, 376 (1- 888 2), $153-160$.

(32) Mello-Vieira, J.; Sousa, T.; Coutinho, A.; Fedorov, A.; Lucas, S. 890 D.; Moreira, R.; Castro, R. E.; Rodrigues, C. M. P.; Prieto, M.; 891 Fernandes, F. Cytotoxic bile acids, but not cytoprotective species, 892 inhibit the ordering effect of cholesterol in model membranes at 893 physiologically active concentrations. Biochim. Biophys. Acta, Biomembr. 894 2013, 1828 (9), 2152-2163.

895

(33) Schubert, R.; Schmidt, K. H. Structural-Changes in Vesicle 896 Membranes and Mixed Micelles of Various Lipid Compositions After 897 Binding of Different Bile-Salts. Biochemistry 1988, 27 (24), 8787- 898 8794.

(34) Nagana Gowda, G. A.; Somashekar, B. S.; Ijare, O. B.; Sharma, 900 A.; Kapoor, V. K.; Khetrapal, C. L. One-step analysis of major bile 901 components in human bile using H-1 NMR spectroscopy. Lipids 2006, 902 41 (6), 577-589. 
904 (35) Fini, A.; Feroci, G.; Roda, A. Acidity in bile acid systems. 905 Polyhedron 2002, 21 (14-15), 1421-1427.

906 (36) De Smet, E.; Mensink, R. P.; Plat, J. Effects of plant sterols and 907 stanols on intestinal cholesterol metabolism: Suggested mechanisms 908 from past to present. Mol. Nutr. Food Res. 2012, 56 (7), 1058-1072. 909 (37) Trautwein, E. A.; Duchateau, G.; Lin, Y. G.; Mel'nikov, S. M.; 910 Molhuizen, H. O. F.; Ntanios, F. Y. Proposed mechanisms of 911 cholesterol-lowering action of plant sterols. Eur. J. Lipid Sci. Technol. 912 2003, 105 (3-4), 171-185.

913 (38) Brown, A. W.; Hang, J.; Dussault, P. H.; Carr, T. P. Phytosterol 914 Ester Constituents Affect Micellar Cholesterol Solubility in Model 915 Bile. Lipids 2010, 45 (9), 855-862.

916 (39) Matsuoka, K.; Hirosawa, T.; Honda, C.; Endo, K.; Moroi, Y.; 917 Shibata, O. Thermodynamic study on competitive solubilization of 918 cholesterol and P-sitosterol in bile salt micelles. Chem. Phys. Lipids 919 2007, 148 (1), 51-60.

920 (40) Matsuoka, K.; Kajimoto, E.; Horiuchi, M.; Honda, C.; Endo, K. 921 Competitive solubilization of cholesterol and six species of sterol/ 922 stanol in bile salt micelles. Chem. Phys. Lipids 2010, 163 (4-5), 397923402.

924 (41) Matsuoka, K.; Rie, E.; Yui, S.; Honda, C.; Endo, K. Competitive 925 solubilization of cholesterol and beta-sitosterol with changing biliary 926 lipid compositions in model intestinal solution. Chem. Phys. Lipids 9272012,165 (1), 7-14.

928 (42) Rozner, S.; Garti, N. The activity and absorption relationship of 929 cholesterol and phytosterols. Colloids Surf., A 2006, 282, 435-456.

930 (43) Hunter, J. E.; Zhang, J.; Kris-Etherton, P. M. Cardiovascular 931 disease risk of dietary stearic acid compared with trans, other saturated, 932 and unsaturated fatty acids: a systematic review. Am. J. Clin. Nutr. 933 2010, 91 (1), 46-63.

934 (44) Brito, R. M. M.; Vaz, W. L. C. Determination of the Critical 935 Micelle Concentration of Surfactants Using the Fluorescent-Probe N936 Phenyl-1-Naphthylamine. Anal. Biochem. 1986, 152 (2), 250-255.

937 (45) Fontell, K. Micellar behaviour in solutions of bile-acid salts. 938 Colloid Polym. Sci. 1971, 246 (1), 614-625.

939 (46) Ijare, O. B.; Somashekar, B. S.; Jadegoud, Y.; Nagana Gowda, G. 940 A. H-1 and C-13 NMR characterization and stereochemical assign941 ments of bile acids in aqueous media. Lipids 2005, 40 (10), 10319421041.

943 (47) Jonsson, B.; Lindman, B.; Holmberg, K.; Kronberg, B. 944 Surfactants and Polymers in Aqueous Solution; John Wiley \& Sons 945 Ltd.: Chichester, England, 1998.

946 (48) Mazer, N. A.; Carey, M. C.; Kwasnick, R. F.; Benedek, G. B. 947 Quasi-Elastic Light-Scattering Studies of Aqueous Biliary Lipid 948 Systems - Size, Shape, and Thermodynamics of Bile-Salt Micelles. 949 Biochemistry 1979, 18 (14), 3064-3075.

950 (49) Melo, E.; Freitas, A. A.; Chang, Y. W.; Quina, F. H. On the 951 significance of the solubilization power of detergents. Langmuir 2001, 95217 (26), 7980-7981.

953 (50) Rozner, S.; Popov, I.; Uvarov, V.; Aserin, A.; Garti, N. 954 Templated cocrystallization of cholesterol and phytosterols from 955 microemulsions. J. Cryst. Growth 2009, 311 (16), 4022-4033.

956 (51) Nielsen, P. B.; Mullertz, A.; Norling, T.; Kristensen, H. G. The 957 effect of alpha-tocopherol on the in vitro solubilisation of lipophilic 958 drugs. Int. J. Pharm. 2001, 222 (2), 217-224.

959 (52) Matsuoka, K.; Nakazawa, T.; Nakamura, A.; Honda, C.; Endo, $960 \mathrm{~K}$.; Tsukada, M. Study of thermodynamic parameters for solubilization 961 of plant sterol and stanol in bile salt micelles. Chem. Phys. Lipids 2008, 962154 (2), 87-93.

963 (53) Richieri, G. V.; Ogata, R. T.; Kleinfeld, A. M. A Fluorescently 964 Labeled Intestinal Fatty-Acid Binding-Protein - Interactions with 965 Fatty-Acids and its use in Monitoring Free Fatty-Acids. J. Biol. Chem. 966 1992, 267 (33), 23495-23501.

967 (54) Ros, E. Intestinal absorption of triglyceride and cholesterol. 968 Dietary and pharmacological inhibition to reduce cardiovascular risk. 969 Atherosclerosis 2000, 151 (2), 357-379. 